DOI: $10.2478 /$ jtam-2013-0004

\title{
NUMERICAL ANALYSIS OF LARGE TELESCOPES IN TERMS OF INDUCED LOADS AND RESULTING GEOMETRICAL STABILITY*
}

\author{
S. Upnere, N. Jekabsons, R. Joffe \\ Engineering Research Institute Ventspils \\ International Radio Astronomy Center of the Ventspils University College, \\ Inzenieru-101, LV-3600, Ventspils, Latvia, \\ e-mails: sabineu@venta.lv, normundsj@venta.lv, robertsj@venta.lv
}

[Received 19 October 2012. Accepted 21 February 2013]

\begin{abstract}
Comprehensive numerical studies, involving structural and Computational Fluid Dynamics (CFD) analysis, have been carried out at the Engineering Research Institute "Ventspils International Radio Astronomy Center" (VIRAC) of the Ventspils University College to investigate the gravitational and wind load effects on large, ground-based radio telescopes RT-32 performance. Gravitational distortions appear to be the main limiting factor for the reflector performance in everyday operation. Random loads caused by wind gusts (unavoidable at zenith) contribute to the fatigue accumulation.

KEY WORDS: Finite Element Method, Computational Fluid Dynamics, large radio telescope, mechanical model.
\end{abstract}

\section{Introduction}

The performance of large ground-based telescopes is directly related to the accuracy of its reflective surfaces. Gravity and wind gusts become dominant sources of surface shape perturbations in the absence of large thermal gradients over the construction, often significantly reducing performance of the whole reflective system. Dominance of the gravitational distortions is expected for a metallic mirror supporting structures. The corresponding deformations are predictable with the aid of Finite Element Method (FEM) application to the structure [1]. On the other hand, wind (in essence turbulent air flow) produces

\footnotetext{
${ }^{*}$ Corresponding author e-mail: sabineu@venta.lv

This research was funded by the European Social Fund (project No. 2009/0231/1DP/ 1.1.1.2.0/09/APIA/VIAA/151).
} 
almost random loads. This may lead to large vibrations of the structure even at moderate and frequent wind velocity [2].

The VIRAC operates two large fully steerable radio telescopes: RT-16 and RT-32 with $16 \mathrm{~m}$ and $32 \mathrm{~m}$ the main reflector diameter, respectively. Both antennas are of Cassegrain type reflecting systems [3].

The RT-32 has become operational again after prolonged delay (19942006) due to transition from military surveillance unit to the radio astronomy site.

Nowadays, it is used for observations such as Very Long Baseline Interferometry, solar observations etc. Notable trait of RT-32 is accurate pointing capabilities and surface adjustment. Accuracy limit by machines estimated by VIRAC engineers is roughly 20" for both - elevation and azimuth. The surface alignment accuracy is well below $1 \mathrm{~mm}$. However, results obtained from observations during 2010-2012 were not according to the expected accuracy and sensitivity of the antenna. For example, no successful low intensity (at $8 \mathrm{GHz}$, below $0.5 \mathrm{Jy}$ ) quasar observations are achieved yet. In order to explain low sensitivity of the antenna, authors carried out studies to determine the influence of external loads on the telescope surface alignment.

The numerical modelling is useful approach to study gravitational [1, 4] and wind loads [5]. In order to perform gravitational stress and strain analysis, FEM beam-based digital model is developed [6]. The Finite Volume Method (FVM) based OpenFOAM solvers are used for solution of differential equations of fluid dynamics. The turbulent phenomenon of air flow is accounted for with Large Eddy Simulation (LES) technique for prediction of dynamical wind loads. For statistical wind load prediction, Reynolds Averaged Navier-Stokes (RANS) equations turbulence models are used [7].

Analysis of the influence of damaged elements on surface accuracy of the radio telescope is another issue addressed by the study. Evaluation of fatigue limiting factors for exploitation life of the construction and reassessment of wind speed limits for different accuracy requirements are still in progress.

\section{Digital model description}

In the absence of technical documentation, direct measurements from actual antenna construction are used for digital model development. However, due to large size and complexity of the object it was impossible to perform all the measurements on site. Some of the parameters (such as wall thickness of beams and plates, locally altered by corrosion) are known only approximately and others have to be assumed based on literature data for the same or similar structures and materials. 
(a)

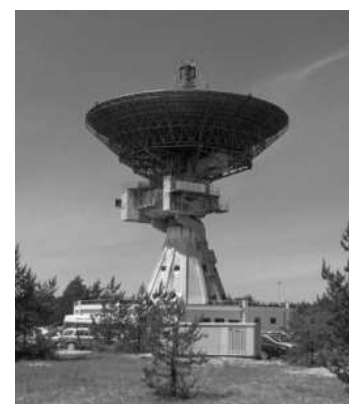

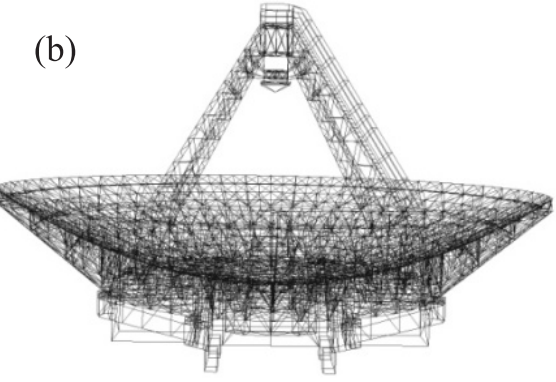

Fig. 1. The photo of RT-32 radio telescope (a) and its digital model (b).

The antenna is mounted on the top of a $25 \mathrm{~m}$ high tower, while the highest point of telescope is about $50 \mathrm{~m}$ above the ground, see Fig. 1(a). The beam-based digital model of the RT-32 dish (Fig. 1(b)) covers surfaces of both mirrors with the supporting construction. The model consists of $\sim 24000$ elements. Beams and plates are used to model the construction [6].

The structure contains damaged elements with corrosion defects which are mapped by visual inspection and included in the model [8]. Digital model of the RT-32 dish is used to describe the corresponding boundary surfaces of the CFD mesh.

\section{lution}

\section{The methodology of wind loads calculations and FEM so-}

In the 3D FVM, model the radio telescope is represented as antenna tower, primary reflector and some of larger beams (Fig. 2(a)). The computation domain is $340 \times 170 \times 134 \mathrm{~m}^{3}$ (length $\times$ width $\times$ height). The $3 \mathrm{D}$ mesh of the computational domain (Fig. 2(b)) is generated by the use of utilities of open source CFD toolkit OpenFOAM. The CFD calculations are performed at three different zenith angles of the primary reflector: $\alpha=0^{\circ}$ (zenith), $60^{\circ}$ and $90^{\circ}$. The Navier-Stokes type equations [9] of incompressible Newtonian fluid are used. Governing equations are solved using OpenFOAM solvers for steady and unsteady flows.

The $k-\varepsilon$ turbulence model with standard transport equations for $k$ and $\varepsilon$ (see [10]) is used for RANS approach, similarly as in [11, 12]. The impact of the wind gusts are evaluated by using scaling factor proportional to the square of the wind speed. Wind measurements indicate that wind gusts are approximately 1.5 times larger than the average wind speed. The model assumes that the radio telescope is located in the atmospheric boundary layer 
(a)

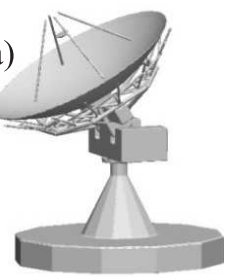

(b)

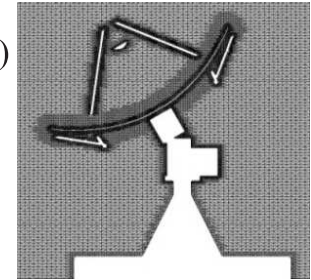

(c)



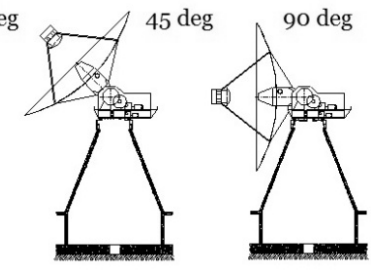

Fig. 2. The FV model of RT-32 (a) and a part of the 3D mesh of computation domain (b). Examples of antenna orientation (c) considered for structural analysis, X-axis points stream wise by respect to wind direction

where wind velocity can be described by logarithmic profile. The temporal average of the wind speed $u_{\text {calc }}(4.6 \mathrm{~m} / \mathrm{s}$ at $50 \mathrm{~m}$ height $)$ from measurements [13] is used to define inlet velocity profile for the CFD modelling. When the wind speed corresponds to a maximum allowable speed for operation of the antenna, $u_{o p}(17.5 \mathrm{~m} / \mathrm{s}$ at $50 \mathrm{~m})$ scaling factor is used.

The structural analysis is carried out by calculations based on the EulerBernoulli beam theory [14]. Standard model with 6 degrees of freedom in every node (three for displacements and rotations) is used. In order to carry out this analysis, in-house FEM software is developed and verified against the commercial FEM package ANSYS. At present only beam type elements are used for analysis and stress values in the beam junctions are not calculated jet. All minor load bearing structures such as steel plates, which form service tunnel or receiver station, are approximated by equivalent beams. Aluminium reflective plates and other structural elements, which bear no considerable mechanical load, are replaced with nodal masses.

The exact type of the steel used in the structure is unknown, but there are indications that it is similar to the AISI 1020 steel with modulus of elasticity $210 \mathrm{GPa}$, Poisson's ratio 0.35 , mass density $7800 \mathrm{~kg} / \mathrm{m}^{3}$, ultimate tensile strength $395 \mathrm{MPa}$, yield strength $295 \mathrm{MPa}$.

For the structural analysis, it is assumed that antenna is loaded only by its own weight. Three loading scenarios, defined by the orientation of antenna (Fig. 2(c)) are considered: 1) vertical (zenith position, $0^{\circ}$ ); 2) rotated by $45^{\circ}$; 3 ) horizontal $\left(90^{\circ}\right)$. The gravitation and wind loads are studied separately. Results of CFD are used to define local forces on a primary reflector (wind load is translated into the nodal structural forces). 
Table 1. The lift $\left(C_{L}\right)$, drag $\left(C_{D}\right)$ coefficients and total force magnitude.

\begin{tabular}{|l|c|c|c|}
\hline Coefficients & $\alpha=0^{\circ}$ & $\alpha=60^{\circ}$ & $\alpha=90^{\circ}$ \\
\hline $\mathrm{C}_{L}$ & 0.779 & -0.647 & 0.004 \\
\hline $\mathrm{C}_{D}$ & 0.463 & 1.052 & 1.073 \\
\hline Total force $[\mathrm{kN}]$ & 17.0 & 118.0 & 117.9 \\
\hline
\end{tabular}

\section{Analysis of the results and discussion}

Comparison of the total wind forces with respect to the different antenna zenith angles is shown in Table 1 . The lift $C_{L}$ and $\operatorname{drag} C_{D}$ coefficients are also calculated (see Table 1 ). The $u_{o p}$ wind speed is used for these calculations. The total force magnitude has maximum at the zenith angle $60^{\circ}$.

The wind induced displacements of a surface of the primary reflector are compared on the circumference of the dish (Fig. 3(a)), since displacements are higher there. The gravitational loads (Fig. 3(b)) cause more significant displacements than wind loads.

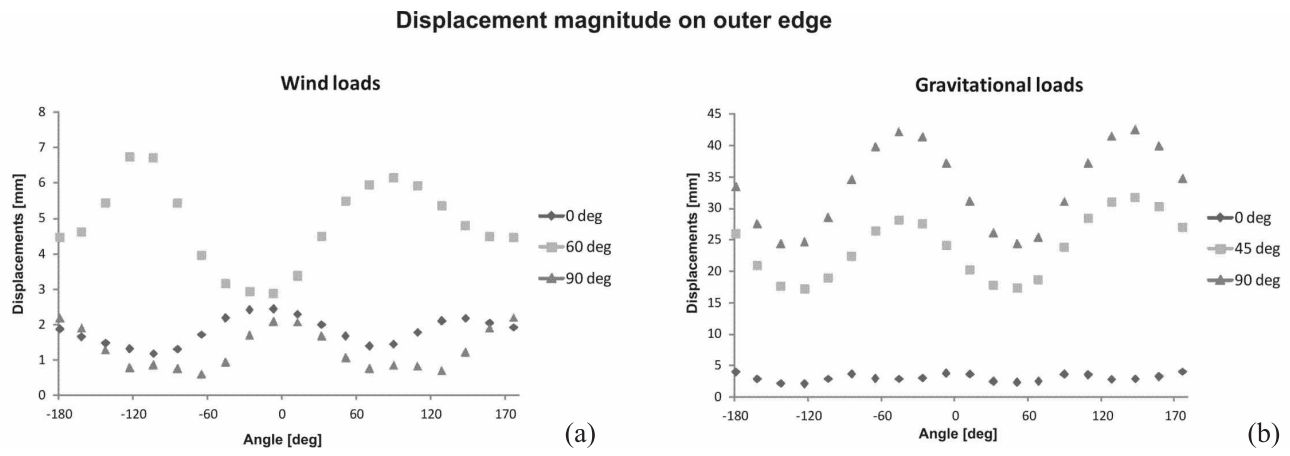

Fig. 3. Displacements on the circumference of the dish caused by wind (a) and gravitational (b) loads.

The displacements due to wind loads reach maximum value of $7 \mathrm{~mm}$ at the zenith angle of $60^{\circ}$. However, the displacement maximum $(\sim 40 \mathrm{~mm})$ for gravitational loads is achieved when zenith angle is $90^{\circ}$. These results are in a good agreement with similar FEM analysis in [4]. In all of these calculations, $u_{o p}$ wind speed is employed.

If errors in the reflector contour are small compared to a wavelength, the relative decrease in aperture efficiency can be expressed according to method- 
ology proposed by Ruze [15]. The comparison of effective area of the reflector for different wavelengths is shown in Fig. 4.

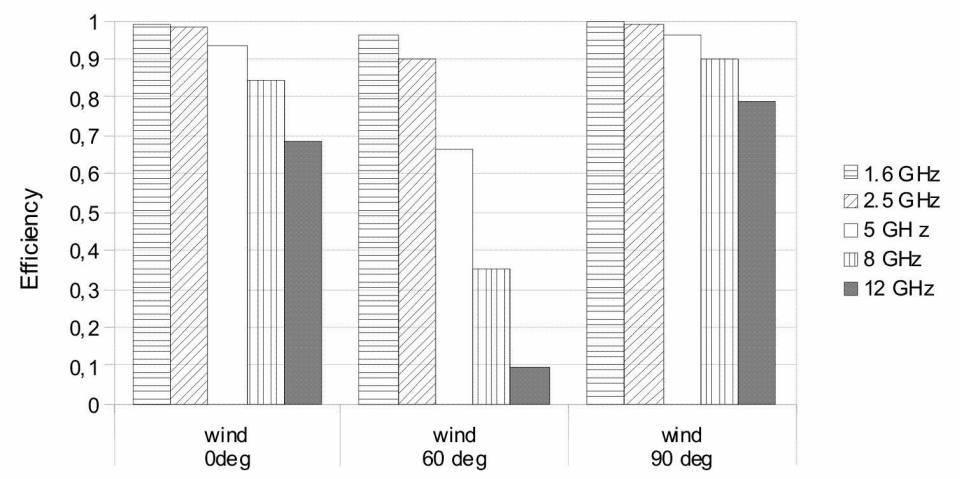

Fig. 4. The effective area of the reflector with respect to different wavelengths.

The maximum Mise stresses in the structural elements at wind speed $u_{o p}$ are estimated to be $20 \mathrm{MPa}$. The wind gusts (frequency $\sim 4000$ times/year) may scale this up till $80 \mathrm{MPa}$. That corresponds to 1,000,000 allowed load cycles (according to the S-N diagram for AISI 1020 steel) [16]. The number of full cycles $\left(0^{\circ}-90^{\circ}\right.$ elevation transitions) is estimated $\sim 4000 /$ year (average operation), or 100,000 per 25 year of exploitation. This leads to $\approx 150 \mathrm{MPa}$ allowed amplitude of average fatigue cycle, which is about one half of maximal effective stress from FEM analysis.

\section{Conclusion}

Digital models were developed and numerical simulations were carried out in order to analyze influence of external environmental factors on radio telescopes.

Gravitational loads dominate for all antenna orientations, inducing deformations of $40 \mathrm{~mm}$ near the horizon, this value fits well with an experimental findings.

The wind load caused deformations by maximal allowed operational wind speeds $(17.5 \mathrm{~m} / \mathrm{s}$ at $50 \mathrm{~m})$ are more moderate than those developing due to gravity. The maximum distortion of the antenna due to wind loads occurs at the zenith angle of $60^{\circ}$ and frontal wind direction. High winds limit operational frequency of antenna to $5 \mathrm{GHz}$, which fits well with the recent 
observations. Under intensive operation critical damage can be accumulated in 25 years. Locations with high stress perturbations can be critical and require additional studies.

\section{REFERENCES}

[1] Woody, D., et al. Measurement, Modelling and Adjustment of the $10.4 \mathrm{~m}$ Diameter Leighton Telescopes, In: Proc. of SPIE, 3357, 1998, 474-485.

[2] Repetto, M. P., G. Solari. Wind-induced Fatigue of Structures under Neutral and Non-neutral Atmospheric Conditions. J. Wind Eng. and Ind. Aerodyn., 95 (2007), 1364-1383.

[3] Balanis, C. A. Antenna Theory. Analysis and Design, New Jersey, John Wiley \& Sons, 2005.

[4] Borovkov, A.I., et al. Finite-Element Modeling and Thermal Analysis of the RT-70 Radio Telescope Main Reflector, In: Proc. of IVth Int. Conf. Antenna Theory and Techniques, 2004, 651-654.

[5] Vogiatzis, K., et al. Estimating the Effect of Wind Loading on Extremely Large Telescope Performance Using Computational Fluid Dynamics, In: Proc. of SPIE, 5497, 2004, 311-320.

[6] Upnere, S., et al. Development of Mechanical Models of RT-16 and RT-32 Radio Telescopes. Space Research Review, 1 (2012), 112-125.

[7] Upnere, S., et al. Characterization of Wind Loading of the Large Radio Telescope. Boundary Field Problems and Computer Simulation, 54 (2012), 30-37.

[8] Joffe, R., et al. Evaluation of Damage and Deformation of the RT-32 Radio Telescope. Latvian J. of Physics and Tech. Sci., 49 (2012), 4-12.

[9] Zienkiewicz, O. C., et al. The Finite Element Method for Fluid Dynamics, Amsterdam, Elsevier BH, 2009.

[10] Launder, B. E., D. B. Spalding. The Numerical Computation of Turbulent Flows. Comput. Method Appl. Mech. Eng., 3 (1974), 269-289.

[11] Upnere, S., et al. Analysis of Wind Influence to Radio Astronomy Observations at Irbene Radio Telescope Complex. Scientific Journal of RTU, 6 (2011), $118-126$.

[12] Upnere, S., et al. Analysis of Structural Integrity of Large Radio Telescopes Subjected to Gravitational and Wind Loads, In: Proc. of EIRI, 25, 2012, 148-154.

[13] ŠIskovs, P., et al. Measurements of the Wind Energy Resource in the Latvia, In: CD Proc. of World Renewable Energy Congress 2011, Sweden, 2011, 1-8.

[14] Zienkiewicz, O. C., R. L. Taylor. The Finite Element Method for Solid and Structural Mechanics, Amsterdam, Elsevier BH, 2006. 
[15] Ruze, J. Antenna Tolerance Theory, a Review, In: Proc. of IEEE, 54, 1966, 633-640.

[16] Beden, S. M., et al. Fatigue Life Assessment of Different Steel-Based Shell Materials under Variable Amplitude Loading. Eur. J. Sci. Res., 29 (2009), $157-169$. 